\title{
Rapid Progression of Scoliosis Requiring Re-Instrumentation after Implant Removal Due to Infection Following Posterior Spinal Fusion and Instrumentation
}

\author{
Keita Nakayama ${ }^{1)}$, Toshiaki Kotani ${ }^{1)}$, Tsuyoshi Sakuma ${ }^{1)}$,Shunji Kishida ${ }^{1)}$, Yuta Muramatsu ${ }^{1)}$, Yu Sasaki ${ }^{1)}$, Keisuke Ueno ${ }^{1)}$ \\ Yasusi Iijima $^{1)}$, Tsutomu Akazawa ${ }^{2)}$, Masashi Yamazaki ${ }^{3)}$ and Shohei Minami ${ }^{1)}$
}

1) Department of Orthopedic Surgery, Seirei Sakura Citizen Hospital, Sakura, Japan

2) Department of Orthopedic Surgery, St Marianna University Hospital, Kawasaki, Japan

3) Department of Orthopedic Surgery, Tsukuba University Hospital, Tsukuba, Japan

\begin{abstract}
Keywords:
scoliosis, posterior spinal fusion and instrumentation, infection, reinstrumentation

Spine Surg Relat Res 2019; 3(1): 102-105 dx.doi.org/10.22603/ssrr.2018-0039
\end{abstract}

An 11-year-old girl was diagnosed with Lenke type 1A Adolescent Idiopathic Scoliosis (AIS). The Cobb angle was $56^{\circ}$, and the Risser grade was 0 (Fig. 1). The patient underwent posterior spinal fusion and instrumentation (PSFI) (T2L2) with pedicle screw and cranial claw hook instrumentation (Fig. 2). Postoperative laboratory data suggested infection. Moreover, she experienced postoperative wound breakdown and a staphylococcus species from the cranial wound, which resolved with antibiotic therapy. A bulge appeared in the skin of her back three months postoperatively because of right cranial rod protrusion. During a second surgery, the right upper rod was cut and the right proximal hooks were removed (Fig. 2). One year after the initial surgery, reddened skin in the cranial wound and an elevated C-reactive protein level suggested infection relapse, which was managed by a third surgery. The partial left rod was cut and the left proximal hooks were removed (Fig. 2). After the third surgery, she did not undergo brace treatment. All three operations were performed in the previous hospital.

Four years after the initial surgery, when the patient was 15 years old, her right thoracic curve had increased to $98^{\circ}$ (Fig. 3). She was referred to our hospital for revision surgery. There were no localized or laboratory findings indicating infection. A radiograph with the patient in a bent position revealed that her main right thoracic curve was fairly rigid (Fig. 3).

A two-stage surgery was planned for the severe rigid scoliosis. All implants were removed during the first surgical stage. On the fused segment at the apex, the lamina was covered with rigid ossification, requiring a Ponte osteotomy. The patient used a maximum $20 \mathrm{~kg}$ halo-wheelchair traction for one month postoperatively, and her right thoracic curve remained rigid with an $85^{\circ}$ angle. During the second surgical stage, an additional osteotomy and corrective revision, consisting of fusion using segmental pedicle screws at T3L3, were performed. After the rod was inserted, motorevoked potentials disappeared from every channel. We adjusted the rods to reduce the correction force, and motorevoked potentials appeared. However, after the patient recovered from anesthesia, both her legs were completely paralyzed.

Nevertheless, one day after surgery, her muscle strength had completely recovered, and she was able to walk within a few days with no signs of neurological abnormality. We used cefotiam $2 \mathrm{~g}$ per day for three days after surgery, and there was no sign of infection. The surgery improved her scoliosis to $63^{\circ}$ (Fig. 4). Moreover, CT after final surgery showed all pedicle screws were appropriately inserted.

Rapid curve progression occurred in this case after partial removal of the implants. Whether or not infection after spine surgery should be treated using implants is controversial. Some studies have reported that implant removal and intravenous administration of antibiotics can silence infection after PSFI ${ }^{1,2)}$. However, some studies reported that removing the implant only led to curve progression after surgery ${ }^{3,4}$ in patients who underwent PSFI and developed late infections.

Corresponding author: Keita Nakayama, keita_nakayama@hotmail.co.jp

Received: June 14, 2018, Accepted: September 14, 2018, Advance Publication: October 19, 2018

Copyright (C) 2019 The Japanese Society for Spine Surgery and Related Research 


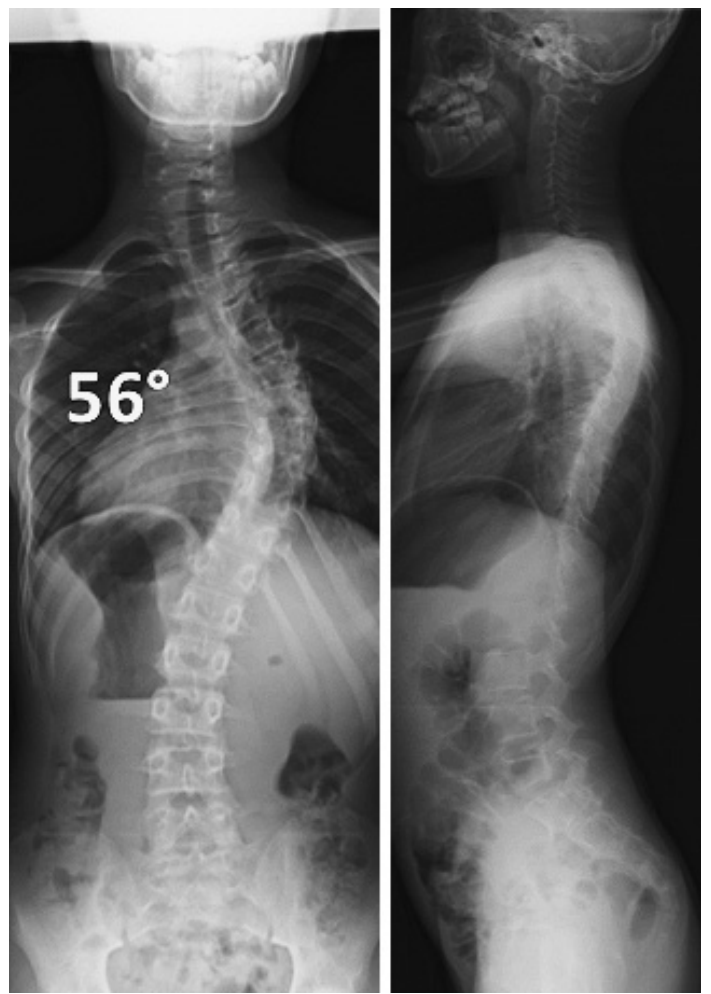

Figure 1. Standing radiographs before initial surgery. The right thoracic Cobb angle was $56^{\circ}$ before her initial surgery.

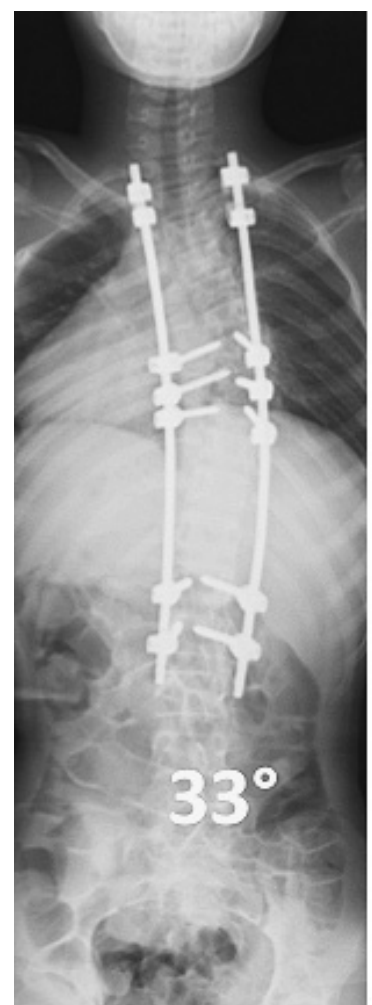

After initial surgery

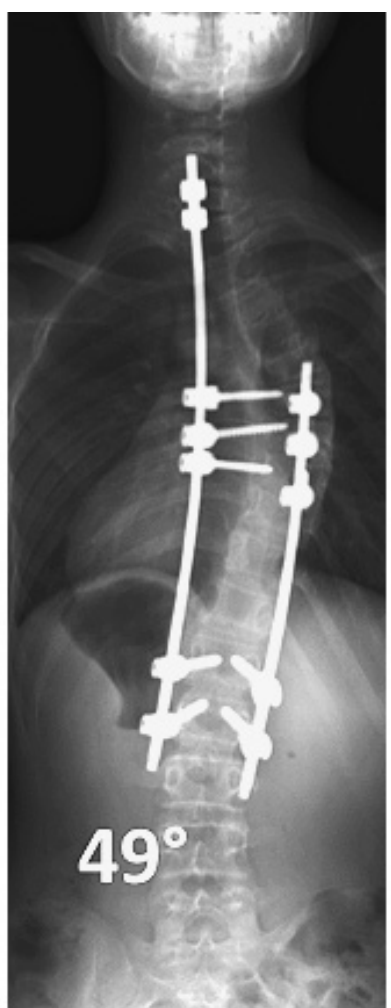

After second surgery

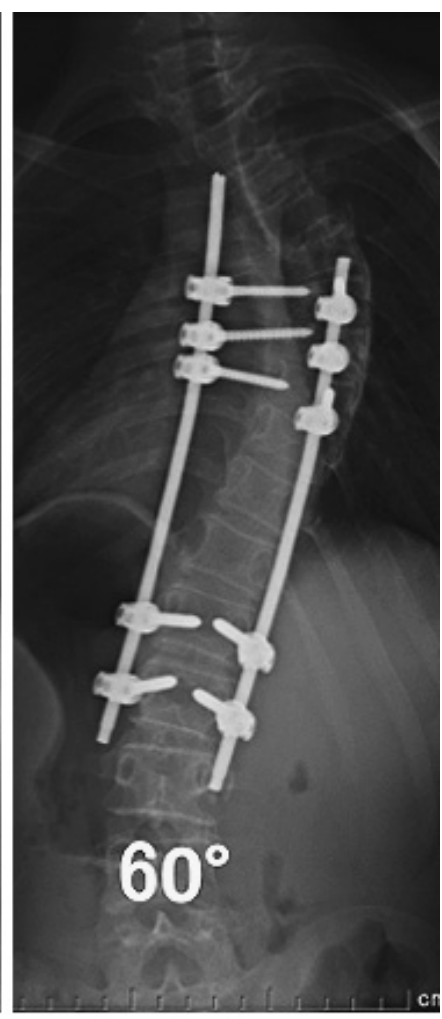

After third surgery

Figure 2. Standing radiographs after initial surgery, second surgery, and third surgery. 


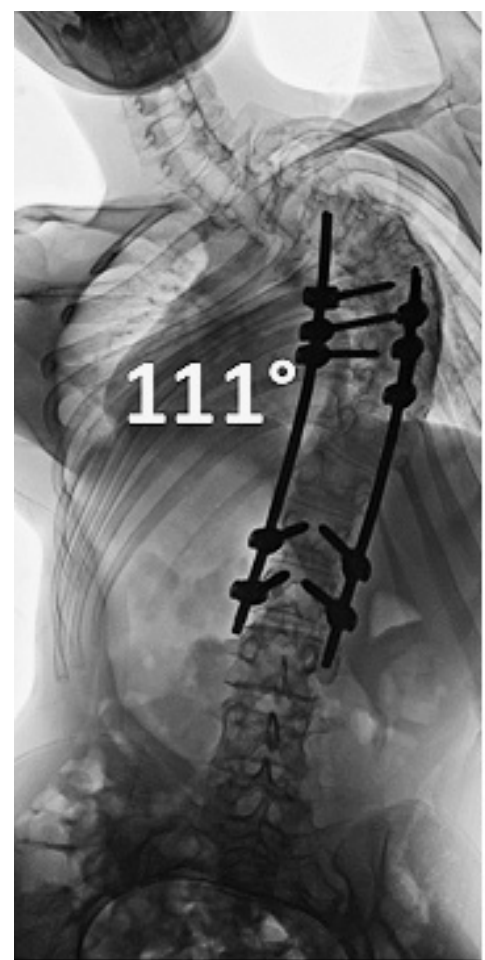

Left bending

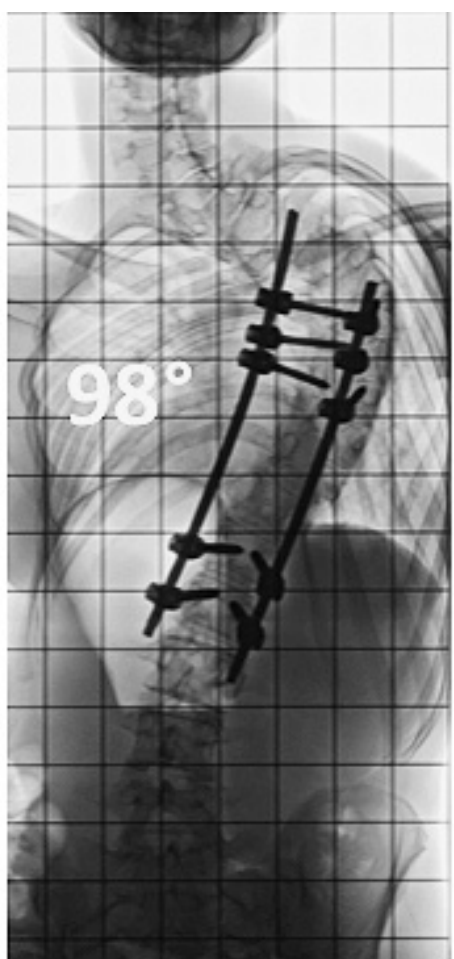

A-P

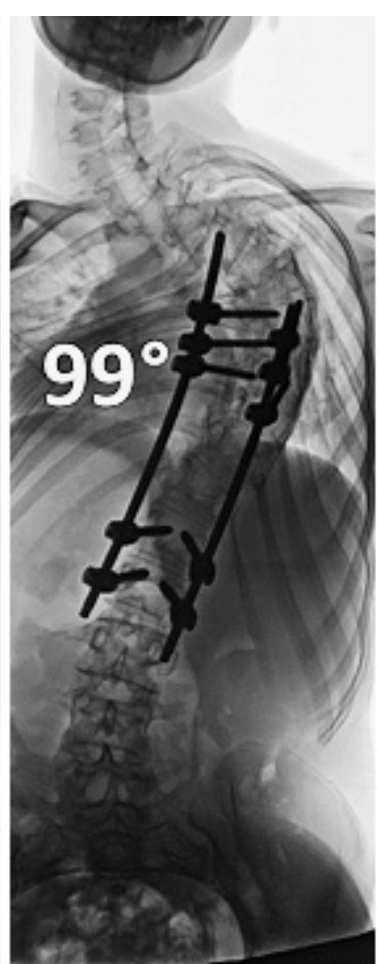

Right bending

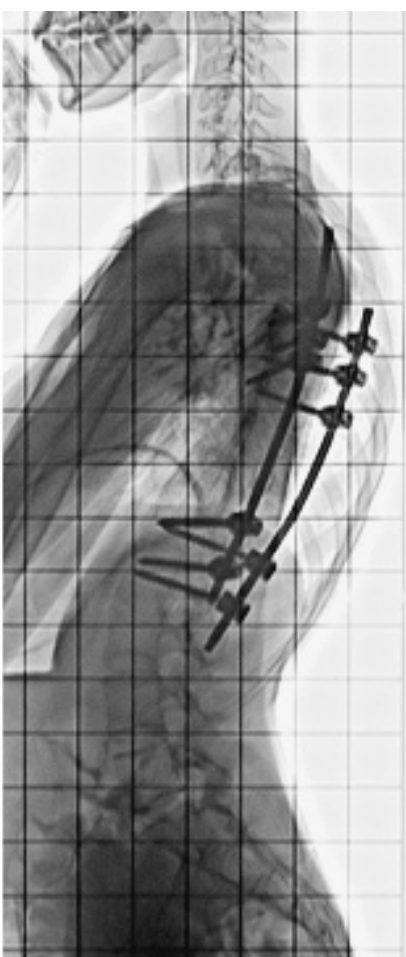

lateral

Figure 3. Standing and bending radiographs prior to reinstrumentation. Four years after the initial surgery, her right thoracic curve had increased to $98^{\circ}$.
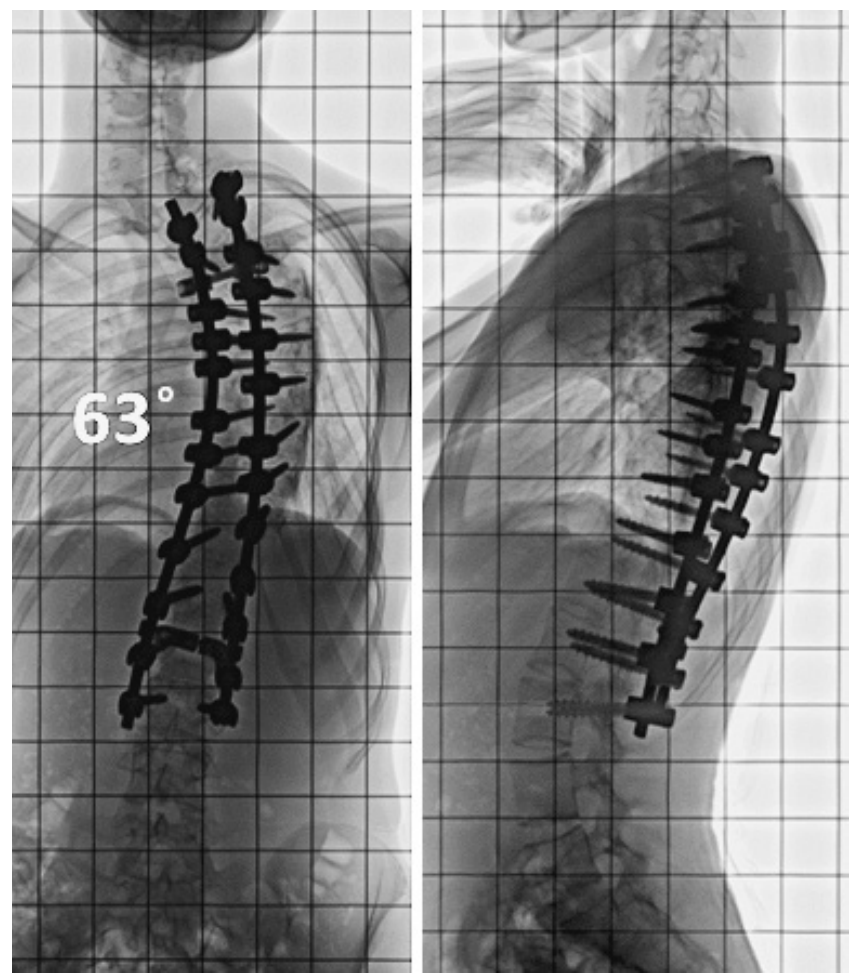

Figure 4. Radiographs after second-stage reinstrumentation surgery. The right thoracic Cobb angle was $63^{\circ}$ after secondstage reinstrumentation surgery.
On the other hand, some reports indicate appropriate debridement and intravenous antibiotic therapy can help patients avoid implant removal ${ }^{5,6}$.

The outcome of our case indicated that debridement delay meant lack of infection control and the patient's inability to retain the implants.

The second important point from our case was the need to perform difficult reinstrumentation surgery because of the rigid curve. Rapid curve progression and a rigid spine requiring osteotomy in the same patient appear to be conflicting situations. Kotani et al., explained the phenomenon by a pseudarthrosis which might have been covered by a weak sheet of bone resembling mature bone fusion ${ }^{3}$.

Our case has led us to make the following important recommendations: 1) we should be aware that rapid curve progression can occur after implant removal; 2) we should explore every available option to help scoliosis patients with infections retain their implants. If we must remove implants to control the infection, we should perform reinstrumentation surgery without delay for patients whose scoliosis progresses.

Conflicts of Interest: The authors declare that there are no relevant conflicts of interest.

Author Contributions: Keita Nakayama wrote and prepared the manuscript, and all of the authors participated in the study design. All authors have read, reviewed, and ap- 
proved the article.

\section{References}

1. Richards BS. Delayed infections following posterior spinal instrumentation for the treatment of idiopathic scoliosis. J Bone Joint Surg Am. 2004;77(4):524-9.

2. Richards BS, Emara KM. Delayed infections after posterior TSRH spinal instrumentation for idiopathic scoliosis: revisited. Spine. 2001;26(18):1990-6.

3. Kotani T, Akazawa T, Lumawig JM, et al. Reinstrumentation for rapid curve progression after implant removal following posterior instrumented fusion in adolescent idiopathic scoliosis: a case report. Scoliosis. 2013;8(1):15

4. Muschik M, Luck W, Schlenzka D. Implant removal for late- developing infection after instrumented posterior spinal fusion for scoliosis: reinstrumentation reduces loss of correction. A retrospective analysis of 45 cases. Eur Spine J. 2004;13(7):645-51.

5. Mirovsky Y, Floman Y, Smorgick Y, et al. Management of deep wound infection after posterior lumbar interbody fusion with cages. J Spinal Disord Tech. 2007;20(2):127-31.

6. Mok JM, Guillaume TJ, Talu U, et al. Clinical outcome of deep wound infection after instrumented posterior spinal fusion a matched cohort analysis. Spine. 2009;34(6):578-83.

Spine Surgery and Related Research is an Open Access journal distributed under the Creative Commons Attribution-NonCommercial-NoDerivatives 4.0 International License. To view the details of this license, please visit (https://creativeco mmons.org/licenses/by-nc-nd/4.0/) 